\title{
Tension modeling of package binding belts in cargo spacecraft
}

\author{
Weifeng Yuan ${ }^{1}$, Hailin Dai ${ }^{1}$ and Ruizhao Du ${ }^{1}$ \\ ${ }^{1}$ Beijing Institute of Spacecraft Environment Engineering, Beijing, China, 100094
}

\begin{abstract}
At the site of cargo package binding in cargo spacecraft, the binding belt tension is loaded with a ratchet device. It is determined by experience of operators, therefore it's difficult to quantify the force and the reliability of the binding belt is low. Based on the dimension analysis method, a semi-quantitative model was established between the binding belt tension and the ratchet force of the fastening device. The motion of the binding process was carefully observed and analyzed based on Analysis of Therbligs. On this basis, the standard packing process is developed, which improves the repeatability of loading and packing pre loading. A loading test platform for cargo loading is built with small tension sensor and tension tester, and a large number of tension data and ratchet force data are obtained. Based on the least-squares linear data fitting, four coupling models for the four binding belts were established. The coupling model verification experiments show that the calculated values are in good agreement with the measured values, and the errors are mostly lower than 5\%. The model can be applied to the quantification of the binding belt tension.
\end{abstract}

The cargo spacecraft is one of the main development models of the space laboratory mission and the space station project in China. It is a necessary support vehicle for the third step long term human care space station. When goods are transported to the space station for supplies, the vast majority of goods are loaded through flexible soft cargo bags. The binding belt is usually used in order to prevent the cargo from slipping and flying during the launch and flight of the cargo ship. The current package binding is carried out manually by the operator, and the tightness of the package binding is also determined by the experience of the operator. Therefore, the consistency of the tightness of the package binding is poor, the pre-loading force is difficult to quantify, and the quality and reliability of the binding cannot be guaranteed, and it is difficult to meet the requirement of quantitative loading for preloading force of cargo binding belt.

The binding belt is made of thick cotton ribbons. In addition to the elastic deformation caused by tension, there is also tension relaxation caused by structural slip and fracture. On one hand, it is more different from the usual elastic body and difficult to predict the binding belt tension by theoretical model; On the other hand, the binding belt is a stereotyped product, and no additional sensors are allowed, so the tension of the binding belt cannot be directly measured. At present, most of the research at home and abroad is focused on tensile fracture performance, safety and comfort of automobile and aircraft passenger seat belts, as well as tension measurement of wire rope, damage mechanism, detection and distribution of stress and strain, etc. However, there is almost no research on the binding of bundles, and no similar research experience and models can be used for reference.

The test platform is set up to simulate the assembly site of the cargo package. The ratchet force and the binding belt tension are measured by the tension sensor connected in the ratchet loading device and the end of the binding belt. On this basis, the coupling model of the 
ratchet force and the binding belt tension is established. In order to improve the repeatability of the coupling model, a set of standard packing process is proposed based on the Analysis of Therbligs. When implementing in the field, operate according to the standard binding process, and predict the binding belt tension according to the ratchet force applied on the ratchet loading device and the coupling model mentioned above. Therefore, the quantitative loading of binding pretension of binding belt can be realized. It has important engineering application value for improving the quality and reliability of package binding.

\section{Cargo package, binding belts and the loading device}

\subsection{Cargo package}

In binding test, the package used is a double package ( length $\times$ width $\times$ height $470 \mathrm{~mm} \times 360 \mathrm{~mm} \times 285 \mathrm{~mm}$, maximum wall thickness $10 \mathrm{~mm}$, maximum weight 0.9 $\mathrm{kg}$, loading capacity $20 \mathrm{~kg}$ ), as shown in Figure 1 .

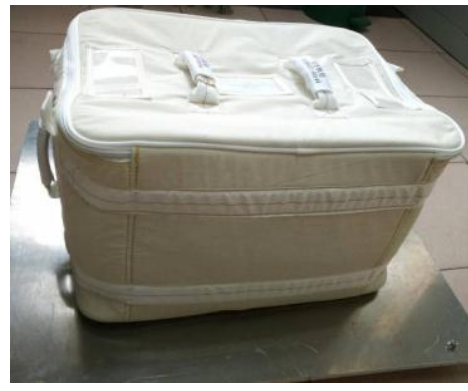

Figure 1. Test package

\subsection{Binding belts}

The belt is made of straw green thick cotton ribbon, as shown in Figure 2.

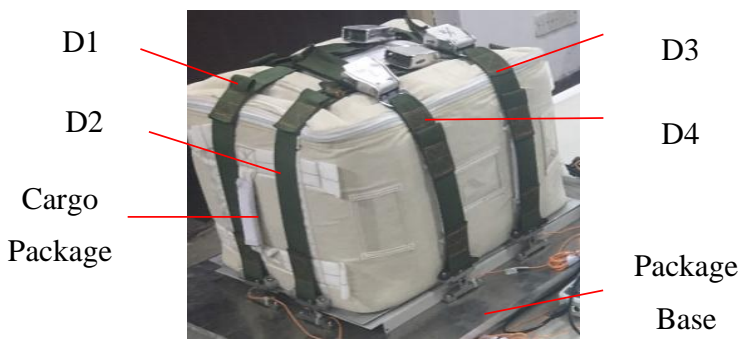

Figure 2. Binding Belts and Package Base

According to the actual working condition of the site, 4 binding bands are sequentially bound according to the order of D2, D4, D1 and D3.

\subsection{Fillings}

Considering that the items in the package are not only hard instruments and equipment, but also soft food and clothing, a flexible paper filler with moderate hardness was selected in the experiment. The filling rate was $95 \%$, leaving an appropriate gap in the interior, and the package could be easily closed.

\subsection{Loading device}

Because of the simple operation, labor-saving and compact size, the ratchet shown in Figure 3 is used as the fastening and loading device for binding belt.

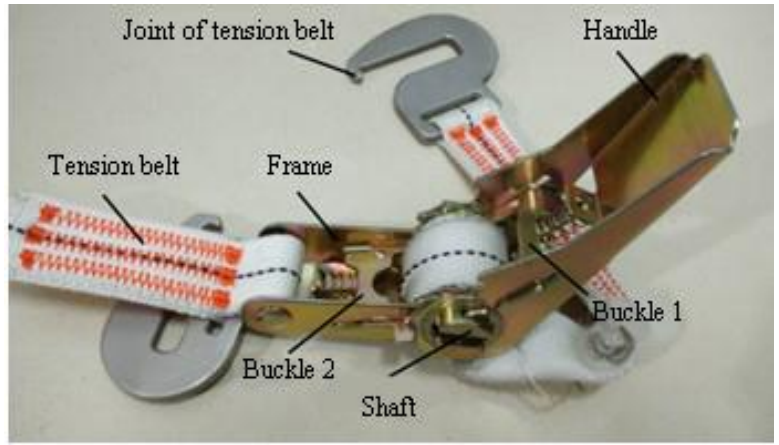

Figure 3. Ratchet fastening and loading device

\section{Analysis of semi-quantitative model of binding belt tension}

\section{1 dimensional analysis}

The binding belt is a stereotyped aerospace product, and the external sensors are not allowed to measure the tension directly. Therefore, it is an effective way to measure the ratchet force imposed by the fastening and loading device, and to predict the binding force through the ratchet force and the binding belt tension coupling model, and then to realize the quantitative loading of the binding belt. In the process of package binding, the binding belt tension is not only related to the size of the ratchet force, but also influenced by the loading speed, the binding method, the safety buckle force and so on. The mechanical properties of the binding belt involve deformation and relaxation, which is very complicated. It is difficult to describe the specific mathematical model For the complex problem with no mathematical model, starting from the basic physics, the semi-quantitative model between the ratcheting force and the binding force can be established by the dimensional analysis, and then 
the function relation of the two is determined by the experiment $^{[1,2,3]}$.

The general formulas are established by selecting the binding belt tensile stress $\sigma\left(\mathrm{kg} /\left(\mathrm{m} \cdot \mathrm{s}^{2}\right)\right)$, the cross section area $S\left(\mathrm{~m}^{2}\right)$, the loading time interval $V$ (times/s), the ratchet force $F_{1}\left(\mathrm{~kg} \cdot \mathrm{m} / \mathrm{s}^{2}\right)$, the safety buckle tension $F_{2}\left(\mathrm{~kg} \cdot \mathrm{m} / \mathrm{s}^{2}\right)$, the binding belt tension $F_{3}\left(\mathrm{~kg} \cdot \mathrm{m} / \mathrm{s}^{2}\right)$ :

$$
f\left(\sigma, S, v, F_{1}, F_{2}, F_{3}\right)=0
$$

Select quality $M$, length $L$ and time $T$ as basic dimensions, and select $\sigma, S, V$ as basic variables. The dimension matrix of the basic variables is

$$
\mathbf{A}=\left[\begin{array}{ccc}
1 & 0 & 0 \\
-1 & 2 & 0 \\
-2 & 0 & -1
\end{array}\right]
$$

The value of the determinant is $|\boldsymbol{A}|=\left|\begin{array}{ccc}1 & 0 & 0 \\ -1 & 2 & 0 \\ -2 & 0 & -1\end{array}\right|=-2 \neq 0$, so $\sigma, S, v$ are linear independent, and can be used as basic variables.

According to the $\pi$ theorem ${ }^{[4,5,6]}$, in the six physical variables $\sigma, S, v, F_{1}, F_{2}, F_{3}$, formulate the dimensionless function of the remaining 3 variables and basic variables respectively $\pi_{1}, \pi_{2}, \pi_{3}$ :

$$
\begin{aligned}
\pi_{1} & =\frac{F_{1}}{\sigma^{x_{1}} S^{y_{1}} v^{z_{1}}} \\
\pi_{2} & =\frac{F_{1}}{\sigma^{x_{2}} S^{y_{2}} v^{z_{2}}} \\
\pi_{3} & =\frac{F_{1}}{\sigma^{x_{3}} S^{y_{1}} v^{z_{3}}}
\end{aligned}
$$

And:

$$
\pi_{3}=f\left(\pi_{1}, \pi_{2}\right)
$$

For $\pi_{1}:\left[\mathrm{MLT}^{-2}\right]=\left[\mathrm{ML}^{-1} \mathrm{~T}^{-2}\right]^{x_{1}}\left[\mathrm{~L}^{2}\right]^{y_{1}}\left[T^{-1}\right]^{z_{1}}(5)$

According to the principle of dimensional harmony:

$$
\begin{gathered}
1=x_{1}, 1=-x_{1}+2 y_{1},-2=-2 x_{1}-z_{1} \\
x_{1}=1, y_{1}=1, z_{1}=0 \\
\pi_{1}=\frac{F_{1}}{\sigma S}
\end{gathered}
$$

Similarly, apply the principle of dimensionless harmony to $\pi_{2}$ and $\pi_{3}$, it can be obtained:

$$
\pi_{2}=\frac{F_{2}}{\sigma S}, \quad \pi_{3}=\frac{F_{3}}{\sigma S}
$$

Formula (8) can be obtained after putting $\pi_{1} \pi_{2}$ and $\pi_{3}$ into formula (4):

$$
F_{3}=f\left(F_{1}, F_{2}\right)
$$

According to formula(8), it can be reached that the output is the preloading force of the binding belt $F_{3}$, and the input is ratchet force $F_{1}$ and the safety buckle force $F_{2}$,which can be measured through tension sensors.
However, it is difficult and inconvenient to fasten the sensor at the end of the binding belt, considering that the measuring device is specially designed to measure the safety buckle force $F_{2}$, Therefore, the safety buckle force can be measured by quantitative control of the descending value of ratchet force.

In this way, the coupling model of binding belt tension $F_{3}$ and safety buckle force $F_{1}$ can be formulated after collecting a large number of data and fitting.

\subsection{Analysis of Therbligs}

Analysis of Therbligs was founded by the American engineer Frank Bunker Gilbreth. The core idea is to establish standardized operation flow through the analysis and reasonable adjustment of the elements of workers' action, so as to reduce the labor intensity and shorten the operation time, and ultimately, to improve the overall efficiency of the operation flow ${ }^{[7,8,9,10,11,12]}$. Therefore, applying Analysis of Therbligs to study the binding process of the cargo package, establishing a standardized operation flow, determining key steps key actions and quantifying can enhance the reproducibility of the binding operation of the cargo package, thereby improving the accuracy of the binding belt tension relaxation model.

Analysis of Therbligs is used to analyze and observe the operation of packing binding. Eight basic actions (Transport Empty, Transport Loaded, grasp, assemble, use, Disassemble, Release Load, Inspect) are selected to describe the whole binding process. On this basis, the operation process is divided into six phases: pre-tightening, pre-loading, step loading, applying safety buckle tension, ratchet unloading and removing ratchet, as shown in Figure 4.

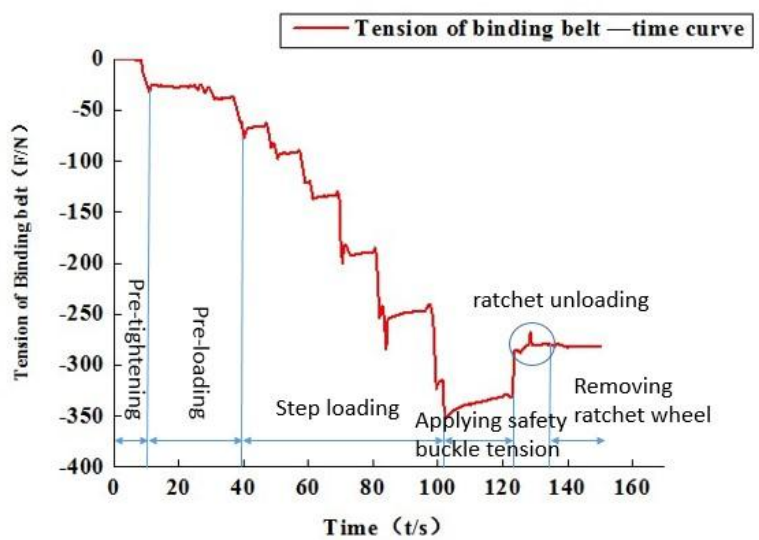


Figure 4. Loading process of binding belts

The six-phase Analysis of Therbligs table is shown in Table 1.

Table 1. Analysis of Therbligs of pre-tightening force of

\begin{tabular}{c} 
package \\
\hline phases \\
applying
\end{tabular}

basic actions pre - pre - step safety ratchet removing tighteningloadingloading buckle unloading ratchet

tension

\begin{tabular}{|c|c|c|c|c|c|c|}
\hline $\begin{array}{c}\text { Transport } \\
\text { Empty }\end{array}$ & $\sqrt{ }$ & $\sqrt{ }$ & $\sqrt{ }$ & $\sqrt{ }$ & $\sqrt{ }$ & $\sqrt{ }$ \\
\hline $\begin{array}{l}\text { Transport } \\
\text { Loaded }\end{array}$ & $\sqrt{ }$ & $\sqrt{ }$ & $x$ & $x$ & $x$ & $\sqrt{ }$ \\
\hline grasp & $\sqrt{ }$ & $\sqrt{ }$ & $\sqrt{ }$ & $\sqrt{ }$ & $\sqrt{ }$ & $\sqrt{ }$ \\
\hline assemble & $\sqrt{ }$ & $\sqrt{ }$ & $x$ & $x$ & $x$ & $x$ \\
\hline use & $x$ & $\sqrt{ }$ & $\sqrt{ }$ & $x$ & $x$ & $x$ \\
\hline $\begin{array}{c}\text { Disassembl } \\
\text { e }\end{array}$ & $x$ & $x$ & $x$ & $x$ & $x$ & $\sqrt{ }$ \\
\hline $\begin{array}{c}\text { Release } \\
\text { Load }\end{array}$ & $\sqrt{ }$ & $\sqrt{ }$ & $\sqrt{ }$ & $\sqrt{ }$ & $\sqrt{ }$ & $\sqrt{ }$ \\
\hline Inspect & $x$ & $\sqrt{ }$ & $\sqrt{ }$ & $\sqrt{ }$ & $x$ & $x$ \\
\hline
\end{tabular}

Note: $\sqrt{ }$ indicates the actions required and $\times$ indicates not.

Through the analysis of each phase, the standard binding process of six-stage binding belt is formulated:

(1)Pre-tightening: tighten the belt safety buckle and the belt, the key action is to make it fit with the package;

(2)Pre-loading: the ratchet loading device is assembled through safety buckles at two ends of the binding belt, and pre-loading is carried out through the ratchet. The key action is to preload the ratchet force $F_{10}$ to $70 \mathrm{~N}$ (the ratchet force is measured by a tension sensor connected in series in the ratchet loading device, as shown in Figure 2 ), and straighten the belt to make it tightly close to the package;

(3)Step loading: pulling the ratchet wheel to load the binding belt, limiting that only one ratchet tooth can be rotated at a time, then straightening the binding belt to make the binding belt fit with the cargo package; Repeat until the ratchet force reaches the predetermined value.

(4)Applying safety buckle tension: hold the binding belt lead and tighten slowly while observing whether the ratchet force $F_{10}$ falls to the specified range $\left(F_{11}\right)$, and then stop.

The optimal value of safety buckle tension obtained through extensive tests is shown in Table 2.

Table 2. The optimal of safety buckle force

\begin{tabular}{cccccc}
\hline $\begin{array}{c}\text { Num } \\
\text { ber }\end{array}$ & $F_{10}$ & $F_{11}$ & Number & $F_{10}$ & $F_{11}$ \\
\hline 1 & -210 & -80 & 7 & -300 & -130 \\
2 & -230 & -90 & 8 & -320 & -135 \\
3 & -250 & -100 & 9 & -340 & -140 \\
4 & -260 & -110 & 10 & -370 & -150 \\
5 & -280 & -120 & 11 & -390 & -160 \\
6 & -290 & -125 & 12 & -400 & -165 \\
\hline
\end{tabular}

Note: define the ratchet force before applying safety buckle force $F_{10}$, the binding belt tension before applying safety buckle force $F_{30}$; the ratchet force after applying safety buckle force $F_{11}$, the binding belt tension after applying safety buckle force $F_{31}$.

(5)Ratchet unloading: press the ratchet loading device buckle to unload the ratchet force.

(6)Remove the ratchet: remove the ratchet hook installed in the belt buckle and remove the ratchet loading device.

\section{Data analysis and modeling}

\subsection{Data acquisition}

Set up the test platform for package binding. The tension sensor is connected in the ratchet loading device and the end of the binding belt, and the ratchet force and the binding belt tension are recorded synchronously by the tension measuring instrument. The tension sensor selects the F2808 micro tension sensor from Tecsis company, matched with the Tecsis B6480 handheld tension tester and the software, as shown in Figure 5.

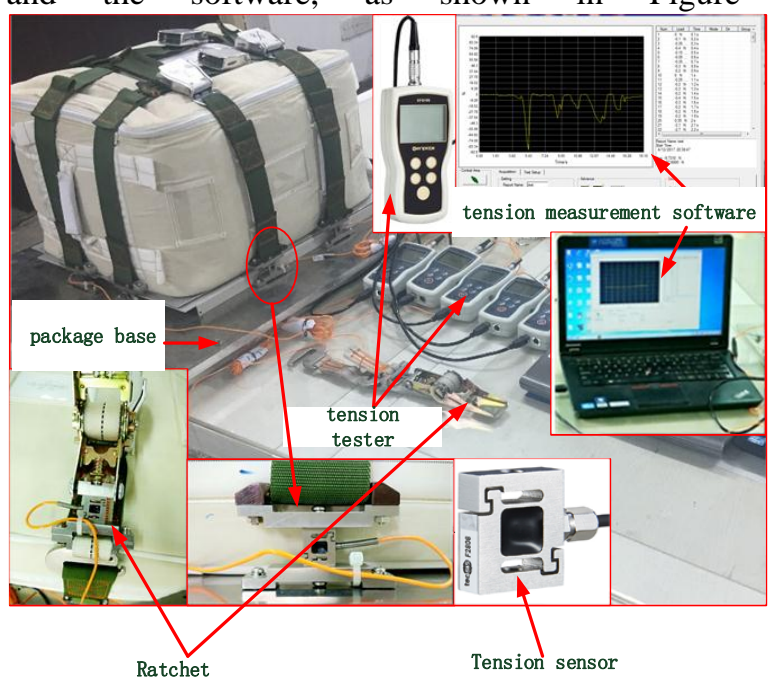

Figure 5. Test platform for package binding

Measurement of ratchet force and binding belt tension is synchronous started in package binding test. A 
series of data of ratchet force $F_{10}, F_{11}$ and binding belt tension $F_{30}$ and $F_{31}$ can be acquired through the software.

\subsection{Steps of coupling modeling}

According to the packing process, the coupling analysis of ratchet force and binding belt tension can be divided into 3 steps:

The first step: establishing the coupling model of $F_{30}$ $-F_{10}$;

The second step: establishing the coupling model of $F_{31}-F_{30}$;

The third step: establishing the coupling model of $F_{31}$ - $F_{10}$ by using function transfer relationship.

During the steps, loading according to the standard binding process obtained by the front element analysis and applying safety buckle tension according to Table 2 are the key point to improve the accuracy of ratchet force and binding belt tension coupling model.

\subsection{Modeling of binding belt tension}

The value of binding belt tension and the ratchet force are in a linear relationship, accordingly a linear data fitting method based on the least square method is used to establish the model ${ }^{[13,14,15]}$.

\subsubsection{Establishing the coupling model of $F_{30^{-}} F_{10}$}

Firstly, the ratchet force data $F_{10}$ and the binding tension data $F_{30}$ obtained from the tension measurement software are used for singular value filtering. Then, select $F_{10}$ as the horizontal axis, the corresponding $F_{30}$ as the longitudinal axis. The $F_{30}-F_{10}$ measured and fitting curves for each binding belt are available through liner fitting, as shown in Figure 6.
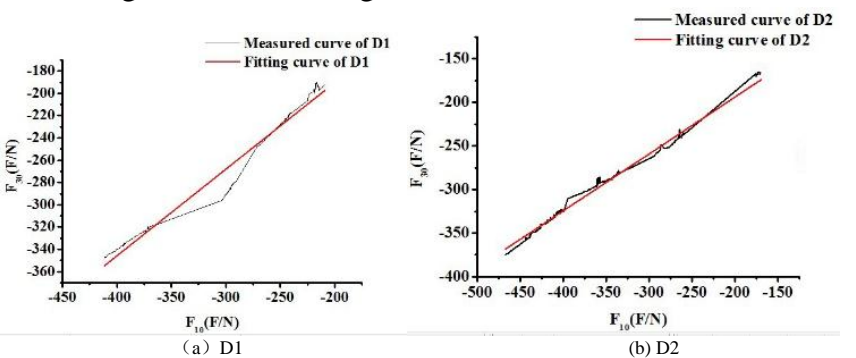
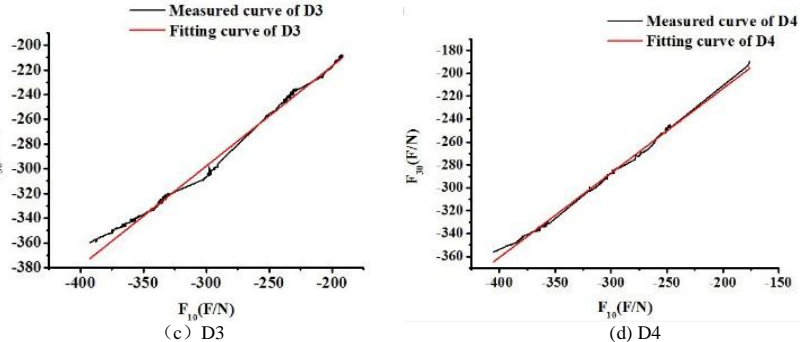

Figure 6. Measured and fitting curve of F30-F10

Corresponding fitting function relations are shown in Table 3:

Table. 3 Coupling formula of $F_{30^{-}} F_{10}$

\begin{tabular}{clc}
\hline Number & linear fitting formula & $\begin{array}{c}\text { Correction } \\
\text { coefficient }\end{array}$ \\
\hline D1 & $F_{30}=-35+0.77736 F_{10}$ & 0.97328 \\
D2 & $F_{30}=-63+0.65327 F_{10}$ & 0.99317 \\
D3 & $F_{30}=-55+0.80969 F_{10}$ & 0.98078 \\
D4 & $F_{30}=-66+0.73751 F_{10}$ & 0.99721 \\
\hline
\end{tabular}

As can be seen from Table 3, the correction coefficient of the fitting curve of the four binding belts of the package is greater than 0.97 , which shows that the fitting degree of $F_{30}-F_{10}$ linear fitting equation is very high and the binding belt tension can be predicted before applying the safety buckle force with a small error.

\subsubsection{Establishing the coupling model of F31 - F30}

Similarly, the binding belt tension before applying the safety buckle force $F_{30}$ is selected as the horizontal axis, the binding belt tension after applying the safety buckle force $F_{31}$ is selected as the longitudinal axis, the scatter plots and fitting lines of each belt are shown in Figure 7.

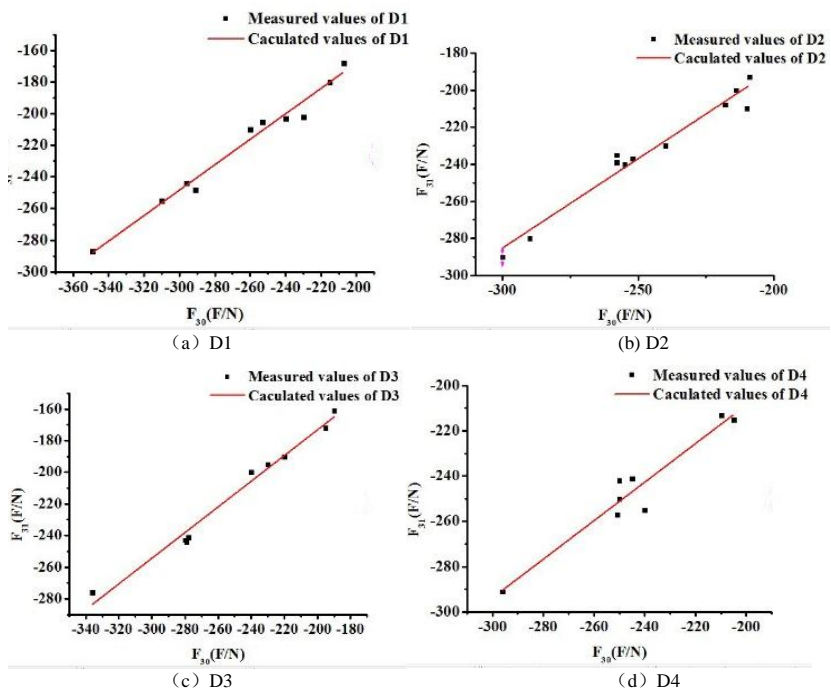

Figure 7. Scatter and fitting curve of $F_{31}-F_{30}$

Corresponding fitting function relations are shown in Table 4.

Table 4. Coupling formula of $F_{31}-F_{30}$ 


\begin{tabular}{ccc}
\hline Number & linear fitting formula & $\begin{array}{c}\text { Correction } \\
\text { coefficient }\end{array}$ \\
\hline D1 & $F_{31}=-10.94+0.81118 F_{30}$ & 0.98005 \\
D2 & $F_{31}=-6.765+0.8051 F_{30}$ & 0.97639 \\
D3 & $F_{31}=-37.93+0.8529 F_{30}$ & 0.91395 \\
D4 & $F_{31}=3.978+0.9636 F_{30}$ & 0.95916 \\
\hline
\end{tabular}

Similarly, as can be seen from Table 4, the correction coefficient of the coupling model of $F_{31}$ and $F_{30}$ is above 0.91 , which is in a high fitting degree.

\subsubsection{Establishing the coupling model of F31-F10}

According to the function transfer relation, the $F_{30}-F_{10}$ fitting equation obtained in Table 3 is brought into the $F_{31}-F_{30}$ fitting equation in Table 4 .And then, the coupling formula of $F_{31}-F_{10}$ can be obtained, as shown in Table 5.

Table 5. Coupling formula of $F_{31}-F_{10}$

\begin{tabular}{cc}
\hline Number & Coupling formula \\
\hline D1 & $F_{31}=-39.33+0.63058 F_{10}$ \\
D2 & $F_{31}=-57.49+0.52595 F_{10}$ \\
D3 & $F_{31}=-84.84+0.69058 F_{10}$ \\
D4 & $F_{31}=-59.62+0.71066 F_{10}$ \\
\hline
\end{tabular}

In this way, according to the coupling model of the ratchet force and the binding belt tension established in Table 5, the binding belt tension can be predicted through the ratchet force measured.

\section{Verification of coupling model}

The measured data of ratchet force under the range of the model are checked and verified by coupling model of $F_{10}$ and $F_{31}$ shown in Table 5. As a result, the scatter plot of calculated $F_{31}$ and measured $F_{31}$ is shown in Figure 8.

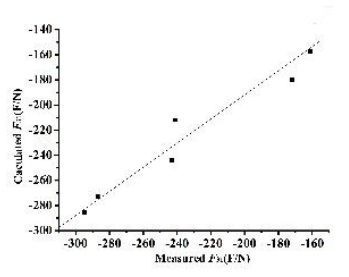

(a) D1

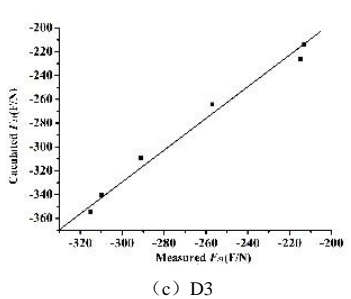

Figure 8. Partial verification result of binding belts tension

As can be seen from Figure 8, most of the calculated values are in good agreement with the measured values, and the errors are mostly less than 5\%. The coupling model of $F_{10}$ and $F_{31}$ can be applied to the quantitative loading in the actual working condition.

\section{Conclusions}

In this paper, the loading of the cargo package binding in cargo spaceship is analyzed deeply. The coupling model of the binding belt tension and ratchet force is established, and the quantitative loading is realized.

1) Based on dimensional analysis, the basic dimensions and basic variables are selected, and a semi-quantitative model between ratchet force and binding belt tension is established.

2) Based on the Analysis of Therbligs, the binding process of cargo package is divided into six phases: pre-tightening, pre-loading, step-loading, applying safety buckle tension, ratchet unloading and ratchet removing. A standard binding process has been established to enhance the repeatability of the binding operation of cargo packages, so as to improve the accuracy of the coupling model of the ratchet force and binding belt tension.

3) The binding test platform is built, which can acquire a lot of ratchet force data and binding belt tension data in real-time; Based on linear regression of least square method, the ratchet force and binding belt tension coupling model of 4 belts were established.

4) The coupling model was checked and verified. The calculated values and measured values were in good agreement, and the errors were mostly less than $5 \%$. Consequently, the coupling model can be applied to the quantitative loading of the cargo package binding in cargo spaceship.

\section{References}

1. Y HU. Discussion about Dimension Analysis and its Application [J].JCJEVC, (2006), 23(2):52-54.

2. Y Y SHAO. Comprehension and Application for Dimensional Analysis and Rule $\pi$ [J].JDIT, (2010), 17(3):106-109.

3. X H XU, J Z HUANG. Application of dimensional analysis in mechanics [J].JMDJU, (2008), 17(1):122-124.

4. B H SUN. Dimensional Analysis and Applications [J].PE, (2016), 26(6):11-20.

5. A G XIE, H NIE. Study on Application Theory of 
the $\pi$ Theorem for Dimension Analysis [J].JASIIST, (2007), 20(5):4-9.

6. Y S DAI, L YANG. Applications of dimensional analysis and the rule $\pi$ in chemical engineering-process improvement of teaching in the course of Principle of Chemical Engineering [J].JNIT, (2010),9(1):11-16.

7. L ZHANG, M CAO,H G PENG, S L ZHOU. Application of therbligs analysis in assembly line balancing [J].JZJUSE, (2010), 22(2):89-94.

8. T GU, B H ZHOU. Application of action analysis method in improvement of cylinder block line balance [J].MM, (2009), 47:54-57.

9. C WU, J HONG, X Y ZHU. Analysis and Improvement on the Operation Process of Turbocharger Assembly Line Based on Therbligs Analysis[J].VE, (2015),20:223-225.
10. X T HU, J ZHAO. The Research of Activity Analysis Design Method Based on the Theory of Motion Analysis [J]. DIMEP, (2011), 24(1):59-61.

11. Y Z ZHU, H F YUAN, L SUN. Analysis by the Color Culture in Huian Women's Costume [J].JCHC, (2015), 17(3):84-90.

12. R Slama, H Wannous, M Daoudi. 3D human motion analysis framework for shape similarity and retrieval [J].IVC, (2014), 32:131-154.

13. Q LIU, Partial Least-squares Regressive Analysis and Modeling for Tool Wear [J].JBHU, (2000), 26(4):457-460.

14. X X SONG. A General Data Fitting Method Based on MATLAB [J].JSXDTU, (2014), 30(4):1-4.

15. L Q ZOU. Least - Square - Multiplication Principle and its Simple Application [J].STI, (2010), 23:282-283 